\title{
Sinonasal Melanoma: A Single Institutional Analysis and Future Directions
}

\author{
Taylor Manton ${ }^{1}$ Brittny Tillman ${ }^{1}$ Jonathan McHugh ${ }^{2} \quad$ Emily Bellile $^{3} \quad$ Scott McLean $^{1} \quad$ Erin McKean ${ }^{1}$
}

${ }^{1}$ Department of Otolaryngology-Head and Neck Surgery, University of Michigan, Ann Arbor, Michigan, United States

2 Michigan Medicine Department of Pathology, Ann Arbor, Michigan, United States

${ }^{3}$ The Center for Cancer Biostatistics, University of Michigan, M2030-28 SPH II, School of Public Health II, Ann Arbor, Michigan, United States

J Neurol Surg B 2019;80:484-492.

\begin{abstract}
Address for correspondence Taylor R. Manton, MD, Department of Otolaryngology-Head and Neck Surgery, University of Michigan, 1904 Taubman Center 1500 East, Medical Center Dr., SPC 5312, Ann Arbor, MI 48109, United States (e-mail: tmanton@med.umich.edu).
\end{abstract}

Abstract

\section{Keywords}

- sinonasal malignancy

- mucosal melanoma

- outcomes

- immunotherapy

- expanded endonasal technique
Background Sinonasal melanoma is a rare disease with a high mortality rate. The surgical management paradigm has significantly changed over the past decade with the introduction of expanded endonasal techniques. There have also been advances in management of metastatic and locally advanced disease with the advent of immunotherapy.

Methods Single-institution retrospective review of adult patients with sinonasal melanoma, surgically managed at the University of Michigan over a 9-year period. Thirty-one patients met inclusion criteria. All patients were retrospectively staged according to the 7th Edition AJCC staging system for mucosal melanoma. Parameters that may affect survival were analyzed using Cox's proportional hazard models and survival outcomes were analyzed with the Kaplan-Meier method. Additionally, a review of three patients with distant metastatic disease receiving immunotherapy is presented.

Results Most patients were managed endoscopically (67\%), and had stage III disease (71\%). However, $57 \%$ of stage IVB tumors were successfully managed endoscopically. Stage statistically impacted overall survival whereas distant control was impacted by stage, site of origin, mitotic rate, and necrosis. The 2-year overall survival for all stages was $77 \%$ which declined with advanced disease. Two-year locoregional control and distant control showed similar trends.

Conclusion Treatment of sinonasal melanoma has drastically changed over the past decade with increased use of expanded endonasal techniques. Our review revealed excellent 2-year overall survival in stage III disease with an appreciable decline in survival in more advanced disease. Immunotherapy may play a large role is future management given the high-risk of distant metastasis.

\section{Introduction}

Sinonasal melanoma is a rare disease entity, accounting for less than $1 \%$ of all diagnosed melanomas and up to $4 \%$ of all malignancies of the sinonasal cavity. ${ }^{1,2}$ It is widely recognized as an aggressive disease that affords a very poor prognosis irrespective of treatment. Standard histopathological predictors of poor prognosis that affect cutaneous melanoma staging,

received

April 3, 2018

accepted after revision

October 6, 2018

published online

December 5, 2018

such as Breslow's depth, ulceration, and mitoses, have not been shown to influence survival in mucosal melanoma. ${ }^{3}$ The published 5-year survival data reveals a dismal rate of 6.5 to $34 \%,{ }^{4}$ with most patients dying within the first 3 years.

The traditional treatment for sinonasal melanoma is surgical resection followed by radiation therapy to improve local control. Surgical management has been shown to afford improved survival outcomes when compared with chemotherapy or

(c) 2019 Georg Thieme Verlag KG Stuttgart · New York
Dol https://doi.org/ 10.1055/s-0038-1676355. ISSN 2193-6331. 
radiotherapy alone. ${ }^{5,6}$ Traditional open approaches are often associated with increased morbidity, prolonged operative times, and significant intraoperative blood loss. Less invasive endoscopic approaches have allowed for decreased morbidity and comparable survival outcomes. ${ }^{7,8}$ Again, the majority of patients undergo adjuvant radiation therapy following surgical resection; however, most studies have shown no significant overall survival benefit. ${ }^{4,9}$

Along with improved surgical techniques, there are now immunologic therapies available for treatment of patients with sinonasal melanoma in the setting of advanced and metastatic disease. These medications include ipilimumab, nivolumab, and pembrolizumab. The data on efficacy of these medications is extrapolated from their use in cutaneous melanoma. ${ }^{10}$ Ipilimumab is a monoclonal antibody which exerts its effect by targeting CTLA-4 (cytotoxic T lymphocyte-associated protein-4). CTLA-4 is a protein receptor which functions by down-regulating the action of cytotoxic T lymphocytes (CTLs) that play a key role in the destruction of tumor cells. Ipilimumab blocks the inhibitory function of CTLA-4 allowing CTL's to perform their tumoricidal role. Nivolumab and pembrolizumab are two antiprogrammed death 1 (PD-1) antibodies which were also approved by the U.S. FDA (Food and Drug Administration) in 2014 for management of metastatic cutaneous melanoma. The therapeutic antibodies bind to and block the PD-1 receptor on lymphocytes inhibiting the PD-L1 and PD-L2 immune suppressing ligands from interacting with the receptor. These ligands are often found on tumor cells which can then bind to T-cells leading to their inactivation resulting in immune evasion. This action is prevented with PD-1 receptor antibodies. These drugs are not yet widely used in the treatment of sinonasal melanoma and the literature regarding their efficacy and safety in this setting is limited. ${ }^{16}$

Given the significant advances in the treatment of this challenging disease entity over the past decade, we elected to review our institutional outcomes and treatment strategies. This study was designed to report the clinical characteristics of patients diagnosed with sinonasal melanoma, analyze the 2year overall survival, locoregional control, distant control and investigate parameters than may be predictive of poor prognosis. We also provide a case report of three patients treated at our facility with advanced sinonasal melanoma receiving immunotherapy, in hopes to shed light on their potential use in the management of this disease in the advanced locoregional and distant metastatic disease setting.

\section{Methods}

An institutional review board approved query of otolaryngology department patient encounters was performed, identifying 183 new patients with nasal or sinonasal malignancy receiving care by the two senior authors between 2009 and 2017. Each patient's chart was examined to include only those patients with biopsy-confirmed sinonasal melanoma, totaling 39 patients. Those patients with metastatic disease at diagnosis $(n=2)$, unresectable disease $(n=2)$ or those whom had undergone surgical resection with curative intent by an outside physician ( $n=4$ ) were excluded, leaving a total of 31 patients for analysis

Clinical information was retrieved from the electronic medical record to include demographic data, presenting symptoms, date of diagnosis, subsite of origin, stage, surgical treatment, complications, adjuvant therapy, and outcome. Each tumor specimen was reviewed by one experienced head and neck pathologist to confirm the diagnosis and identify histopathological features that may be associated with prognosis. Each patient was retrospectively staged according to the 7th Edition American Joint Committee on Cancer (AJCC) staging system for mucosal melanoma ( Table $\mathbf{1}$ ) based on clinical information including endoscopic description, radiologic data, and intraoperative findings. At the time of this publication, the AJCC 8th edition has been released with no changes in the tumor-node-metastatis (TNM) staging; however, there are now no proposed prognostic stage groups.

Outcome data pertaining to patient and disease parameters were calculated using Cox proportional hazards models for 2year overall survival(OS), locoregional control(LC), and distant control (DC). Curves describing the 2-year OS, LC, and DC were calculated with the Kaplan-Meier method.

During our review, three patients were identified who had received immunotherapy. Clinical data was collected regarding tumor stage, primary site, date of diagnosis and surgery, operative technique, receipt of adjuvant radiation, site of recurrence/distant metastasis, immunotherapy regimen, and outcomes.

Table 1 AJCC 7th edition staging for mucosal melanomas

\begin{tabular}{|l|l|}
\hline \multicolumn{2}{|l|}{$\begin{array}{l}\text { Description of the 7th edition AJCC staging system } \\
\text { for sinonasal melanoma }\end{array}$} \\
\hline T & Primary tumor \\
\hline T3 & Mucosal disease \\
\hline T4a & $\begin{array}{l}\text { Moderately advance disease: tumor involving } \\
\text { deep soft tissue, cartilage, bone, or overlying skin }\end{array}$ \\
\hline T4b & $\begin{array}{l}\text { Very advance disease: tumor involving brain, dura, } \\
\text { skull base, lower cranial nerves (IX, X, XI, XII), } \\
\text { masticator space, carotid artery, prevertebral } \\
\text { space, or mediastinal structures }\end{array}$ \\
\hline N & Regional lymph nodes \\
\hline Nx & Regional lymph nodes cannot be assessed \\
\hline N0 & No regional lymph node metastasis \\
\hline N1 & Regional lymph node metastasis present \\
\hline M & Distant metastasis \\
\hline M0 & No distant metastasis \\
\hline M1 & Distant metastasis \\
\hline Overall stage \\
\hline III & T3 N0 M0 \\
\hline IVA & $\begin{array}{l}\text { T4a N0 M0 } \\
\text { T3-4a N1 M0 }\end{array}$ \\
\hline IVB & T4b any N M0 \\
\hline IVC & Any T any N M1 \\
\hline
\end{tabular}




\section{Results}

\section{Patient and Disease Characteristics}

A total of 31 patients met inclusion criteria, most of whom were female, Caucasian, and nonsmokers with greater than 60 years of age. The most common site of disease origin was within the nasal cavity, specifically arising from the nasal septum. Most patients presented with multiple symptoms including: epistaxis, nasal obstruction, sinusitis, and cranial neuropathies. Less common symptoms included headache, nasal deformity, otalgia, hearing loss, facial pain, vision changes, and epiphora. All stages of disease were represented in this cohort, the most common being stage III. Disease and patient characteristics are summarized in -Table 2 .

Table 2 Patient demographics, disease characteristics and pathologic variables

\begin{tabular}{|c|c|}
\hline Patient demographics & Number (\%) $n=31$ \\
\hline Age in y (mean and range) & $71.0(52-85)$ \\
\hline$<60$ & $6(19)$ \\
\hline$>60$ & $25(81)$ \\
\hline \multicolumn{2}{|l|}{ Race } \\
\hline White & $29(93)$ \\
\hline Black & $2(7)$ \\
\hline Other & $0(0)$ \\
\hline \multicolumn{2}{|l|}{ Gender } \\
\hline Male & $9(29)$ \\
\hline Female & $22(71)$ \\
\hline \multicolumn{2}{|l|}{ Tobacco use } \\
\hline Yes & $12(39)$ \\
\hline No & $19(61)$ \\
\hline \multicolumn{2}{|l|}{ Disease characteristics } \\
\hline \multicolumn{2}{|l|}{ Primary site } \\
\hline Nasal cavity & $23(75)$ \\
\hline Paranasal sinuses & $8(25)$ \\
\hline \multicolumn{2}{|l|}{ AJCC overall stage } \\
\hline III & $19(61)$ \\
\hline IVA & $5(16)$ \\
\hline IVB & $7(23)$ \\
\hline \multicolumn{2}{|c|}{ Time from diagnosis to surgery } \\
\hline$<1 \mathrm{mo}$ & $11(35)$ \\
\hline $1-2 \mathrm{mo}$ & $15(48)$ \\
\hline$>2 \mathrm{mo}$ & $5(16)$ \\
\hline mean (range) & $42.8 \mathrm{~d}(8-190)$ \\
\hline \multicolumn{2}{|l|}{ Presenting symptoms } \\
\hline Epistaxis & $27(87)$ \\
\hline Nasal obstruction & $17(55)$ \\
\hline
\end{tabular}

Table 2 (Continued)

\begin{tabular}{|c|c|}
\hline Patient demographics & Number (\%) $n=31$ \\
\hline Sinusitis & $6(19)$ \\
\hline Cranial neuropathy & $3(10)$ \\
\hline Pathologic variables & Number $(\%) n=22$ \\
\hline \multicolumn{2}{|l|}{ Anatomic level } \\
\hline Bone & $7(37)$ \\
\hline Submucosa & $14(64)$ \\
\hline Not available & $1(5)$ \\
\hline Ulceration & $14(64)$ \\
\hline Necrosis & $9(41)$ \\
\hline Satellitosis & $2(9)$ \\
\hline Perineural invasion & $1(5)$ \\
\hline Angiolymphatic invasion & $2(9)$ \\
\hline \multicolumn{2}{|l|}{ Pigmentation } \\
\hline Absent & $12(55)$ \\
\hline Extensive & $7(32)$ \\
\hline Focal & $3(14)$ \\
\hline \multicolumn{2}{|l|}{ Subtype } \\
\hline Epitheliod & $16(73)$ \\
\hline Small cell & $4(18)$ \\
\hline \multirow[t]{2}{*}{ Spindle } & $2(9)$ \\
\hline & Mean (SD), range \\
\hline Mitosis (per $\mathrm{mm}^{2}$ ) & $6.9(6.4), 0-18$ \\
\hline Depth (mm) & $7.0(6.4), 0-25$ \\
\hline
\end{tabular}

Abbreviation: SD, standard deviation.

\section{Pathologic Characteristics}

All retained pathologic specimens were submitted for internal histological review for confirmation of diagnosis. Of the 31 patients, 16 had BRAF and cKIT mutation status available for interpretation. Of these 16 patients, only 3 were positive for cKIT and 1 was positive for BRAF mutations. Twenty-two samples had sufficient tissue available for further analysis of pathologic features that may influence clinical outcomes. These variables are listed in - Table 2. On univariate analysis, mitotic rate (analyzed as a continuous variable), necrosis, and anatomic level within bone compared with submucosa each had a statistically significant decreased time to develop distant disease with hazard ratios of 1.16 (95\% CI [confidence interval]: $1.02-1.33) p=0.03,9.48$ (95\% CI: $1.76-51.24)$ $p=0.009$, and 6.41 (95\% CI: 1.19-34.43) $p=0.03$, respectively. In multivariate analysis, mitotic rate and necrosis separately add information to our DC model over stage and disease site; however, anatomic level loses significance after controlling for these variables. These outcomes are displayed in -Table 3.

\section{Treatment}

Surgical intervention was categorized by approach including endoscopic, open, or combined. Twenty-one patients (67\%) 
Table 3 Hazard ratios and p-values from Cox's proportional hazards models for overall survival (OS), locoregional control (LC), and distant control (DC)

\begin{tabular}{|l|l|l|l|}
\hline & OS & LC & DC \\
\hline Depth $(\mathrm{mm})$ & $0.935(0.689,1.270), p=0.67$ & $0.966(0.766,1.218), p=0.77$ & $1.113(0.993,1.247), p=0.065$ \\
\hline Mitoses $\left(\mathrm{mm}^{2}\right)$ & $1.093(0.969,1.233), p=0.15$ & $1.032(0.912,1.166), p=0.62$ & $1.164(1.016,1.334), p=0.03$ \\
\hline Ulcerated & $1.248(0.278,5.615), p=0.77$ & $1.248(0.278,5.615), p=0.88$ & $5.709(0.679,48.038), p=0.11$ \\
\hline Necrosis & $p=0.9966, \mathrm{HR}$ est. problems & $0.696(0.077,6.308), p=0.75$ & $9.483(1.755,51.248), p=0.009$ \\
\hline Satelitosis & $1.251(0.149,10.507), p=0.84$ & $4.139(0.374,45.763), p=0.25$ & $1.450(0.173,12.128), p=0.73$ \\
\hline PNI & $4.500(0.456,44.374), p=0.20$ & $5.283(0.545,51.175), p=0.15$ & $p=0.9954$, HR est. problems \\
\hline ALI & $6.380(0.564,72.180), p=0.13$ & $p=0.9959$, HR est. problems & $7.159(0.636,80.576), p=0.11$ \\
\hline $\begin{array}{l}\text { Anatomic level } \\
\text { (bone vs. } \\
\text { submucosa) }\end{array}$ & $1.960(0.347,11.059), p=0.45$ & $0.387(0.045,3.329), p=0.39$ & $6.407(1.192,34.426), p=0.03$ \\
\hline $\begin{array}{l}\text { Pigmentation } \\
\text { (extensive vs. } \\
\text { absent) }\end{array}$ & $0.448(0.086,2.327), p=0.34$ & $0.160(0.017,1.541), p=0.11$ & $1.175(0.235,5.875), p=0.84$ \\
\hline $\begin{array}{l}\text { Pigmentation } \\
\text { (focal vs. absent) }\end{array}$ & $p=0.9963, \mathrm{HR}$ est. problems & $2.879(0.249,33.326), p=0.40$ & $0.976(0.101,9.458), p=0.98$ \\
\hline $\begin{array}{l}\text { Subtype } \\
\text { (small cell vs. } \\
\text { epitheloid) }\end{array}$ & $2.790(0.544,14.310), p=0.22$ & $4.268(0.620,29.402), p=0.14$ & $2.017(0.368,11.072), p=0.42$ \\
\hline $\begin{array}{l}\text { Subtype } \\
\text { (spindle vs. } \\
\text { epitheloid) }\end{array}$ & $1.306(0.132,12.899), p=0.82$ & $2.807(0.290,27.128), p=0.37$ & $1.958(0.218,17.605), p=0.55$ \\
\hline
\end{tabular}

Abbreviations: ALI, angiolymphatic invasion; HR, hazard ratio; PNI, perineural invasion.

were treated endoscopically, seven (23\%) were treated with a combined approach, and three (10\%) via an open approach. Those treated endoscopically encompassed all disease stages with 15 patients representing stage III, two patients (40\%) stage IVA and four patients (57\%) stage IVB. Those patients treated with a combined approach also encompassed all disease stages, with four stage III, two stage IVA, and one stage IVB. Open approach was reserved for advance disease alone including for one stage IVA and three stage IVB. Most patients went on to receive adjuvant radiation therapy at $84 \%$. Of the five patients who did not receive adjuvant radiation therapy, four had disease limited to the mucosa with negative surgical margins. Two of these patients had no residual melanoma found within the surgical specimen, owing to their initial biopsy having removed all malignant cells. Each patient also had a Multidisciplinary Melanoma Tumor Board recommendation against additional therapy, given the limited extent of disease. The fifth patient presented with stage IVB disease and developed distant metastatic disease within 2 weeks of surgical resection, succumbing to the disease within 5 months. Adjuvant radiation therapy was initially recommended but held due to initiation of systemic immunotherapy for management of distant disease.

We thus far have three patients on immunotherapy for the treatment of distant metastatic disease. Treatment regimens include: ipilimumab alone, ipilimumab with nivolumab, and pembrolizumab alone. Details regarding these cases are described later. Treatment data are summarized in - Tables 4 and $\mathbf{5}$.

\section{Survival}

The median follow-up duration was 38.5 months. The 2-year overall survival (OS) for all stages was 77\% (95\% CI: 56-

Table 4 Treatment rendered

\begin{tabular}{|l|l|}
\hline Treatment & Number (\%) \\
\hline Total patients & $n=31$ \\
\hline Surgical modality & \\
\hline Endoscopic & $21(67)$ \\
\hline Open & $3(10)$ \\
\hline Combined & $7(23)$ \\
\hline Margin status & \\
\hline Positive & $8(26)$ \\
\hline Endoscopic & $6(75), 28 \%$ of all endoscopic \\
\hline Combined & $1(12.5) 14 \%$ of all combined \\
\hline Open & $1(12.5), 33 \%$ of all open \\
\hline Negative & $23(74)$ \\
\hline Adjuvant XRT & \\
\hline Yes & $26(84)$ \\
\hline No & $5(16)$ \\
\hline Immunotherapy & \\
\hline Yes & $3(10)$ \\
\hline No & $28(90)$ \\
\hline
\end{tabular}

Abbreviations: XRT, radiation. 
Table 5 Surgical technique by stage

\begin{tabular}{|l|l|}
\hline Surgical technique by stage & Number (\%) \\
\hline Stage III & $n=19(61)$ \\
\hline Endoscopic & $15(79)$ \\
\hline Combined & $4(21)$ \\
\hline Open & $0(0)$ \\
\hline Stage IVA & $n=5(16)$ \\
\hline Endoscopic & $2(40)$ \\
\hline Combined & $2(40)$ \\
\hline Open & $1(20)$ \\
\hline Stage IVB & $n=7(23)$ \\
\hline Endoscopic & $4(57)$ \\
\hline Combined & $1(14)$ \\
\hline Open & $2(29)$ \\
\hline
\end{tabular}

89; - Fig. 1), locoregional control (LC) was 72\% (95\% CI: 4986), distant control (DC) was 69\% (95\% CI: 47-83). When subdivided by stage, the 2-year OS was $87 \%$ (95\% CI: $58-97$ ), 67\% (95\% CI: 5-95) and 57\% (95\% CI: 17-84) for stages III, IVA, and IVB, respectively (-Fig. 2). The 2-year LC was 72\% (95\% CI: 62-97), 100\% (no local recurrences), and 38\% (95\% CI: $1-$ 81 ) for stages III, IVA, and IVB, respectively (-Fig. 3). The 2- year DC was 89\% (95\% CI: 62-97), 33\% (95\% CI: 1-77), and 21\% (95\% CI: 1-60) for stages III, IVA, and IVB, respectively (-Fig. 4). These outcomes are summarized in -Table 6. Stage was the only variable found to significantly impact OS with a hazard ratio of 3.87 (95\% CI: 1.02-14.74) when comparing stage III disease to stage IVB. However, DC was significantly impacted by both stage and primary site of tumor origin within the nasal cavity. Hazard ratios were 7.65 (95\% CI: 1.51-38.71) for stage IVA compared with stage III and 7.38 (95\% CI: 1.56-34.84) for stage IVB compared with stage III disease. For tumor origin, the hazard ratio was 6.06 (95\% CI: 1.58-23.27) for the nasal cavity when compared with the paranasal sinuses. Cox's proportional hazards models for outcome measures are displayed in - Table 7.

\section{Future Directions of Management: Utilization of Immunotherapy}

Three postsurgical patients have received immunotherapy in our cohort. The first patient had T4bNOM0 mucosal melanoma of the ethmoid sinus and underwent an open approach for tumor resection followed by adjuvant radiation therapy. They developed multiple distant metastases including pelvic lymph nodes and a temporal lobe metastasis, first identified 16 months following surgery. Immunotherapy with combined ipilimumab and nivolumab was initiated at the time of diagnosis of distant disease. This patient remains with stable

\section{2-Year overall survival}

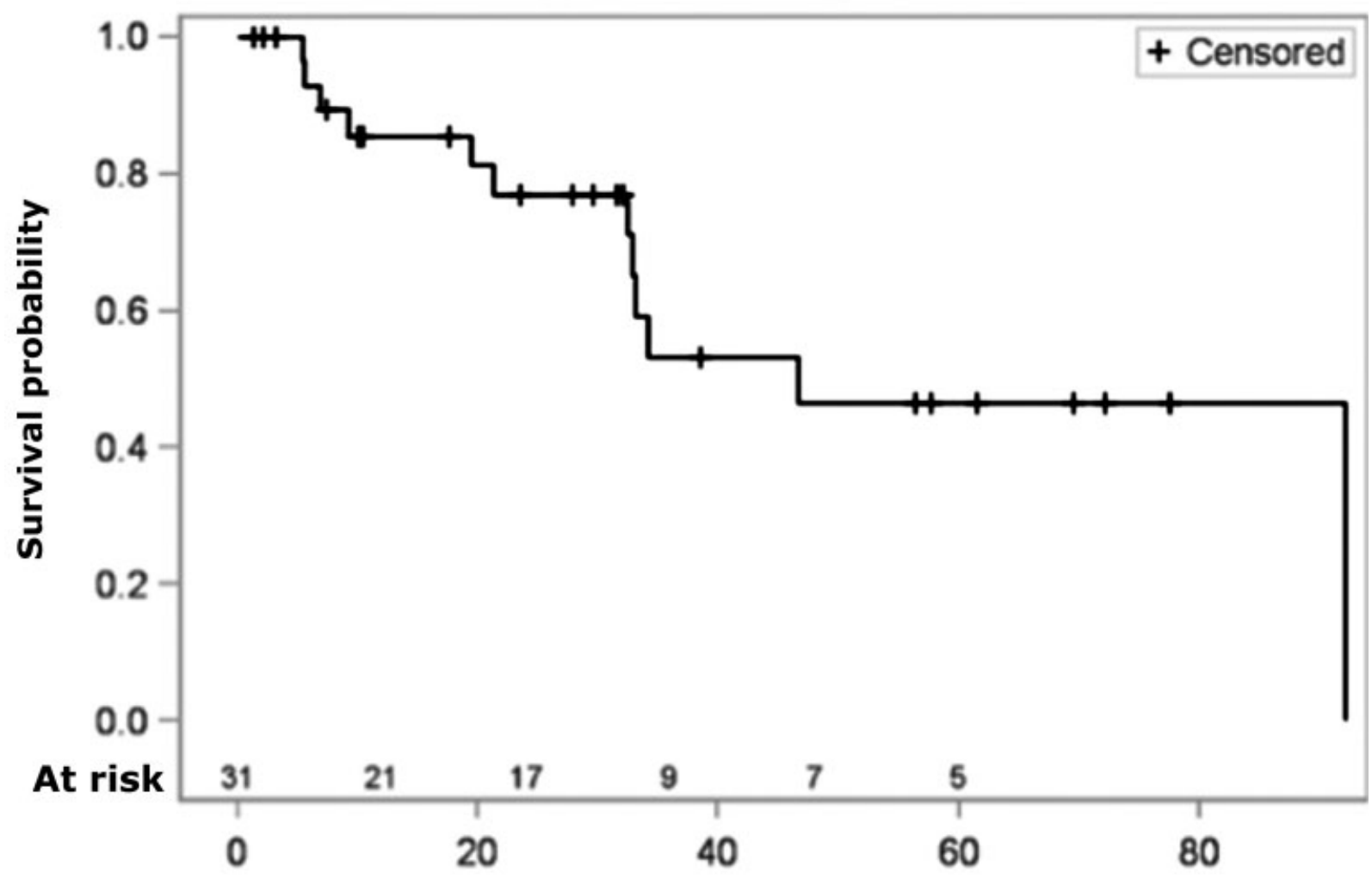

Months from surgical resection

Fig. 1 2-year overall survival for all stages. 


\section{2-Year overall survival by stage}

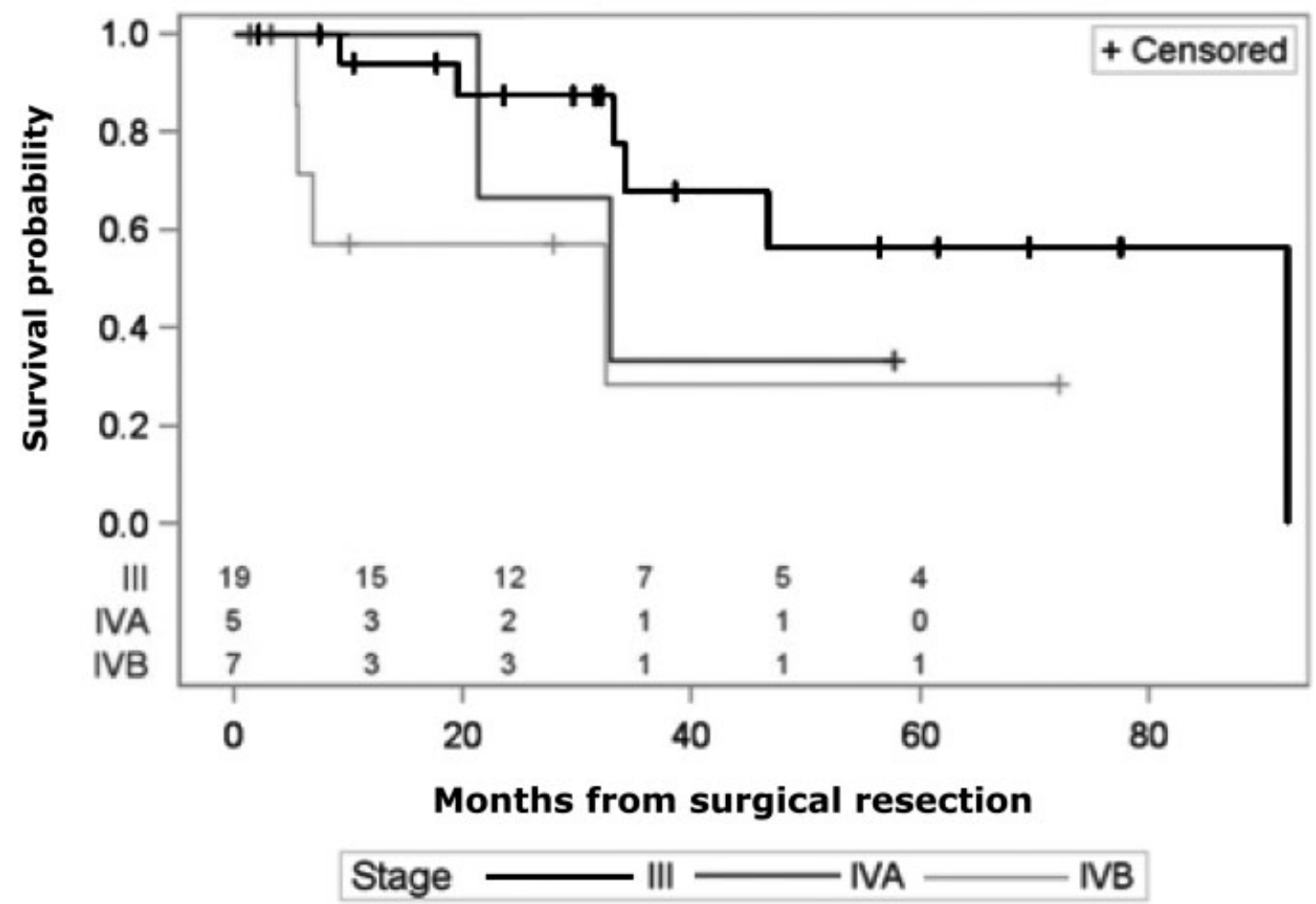

Fig. 2 2-year overall survival by stage.

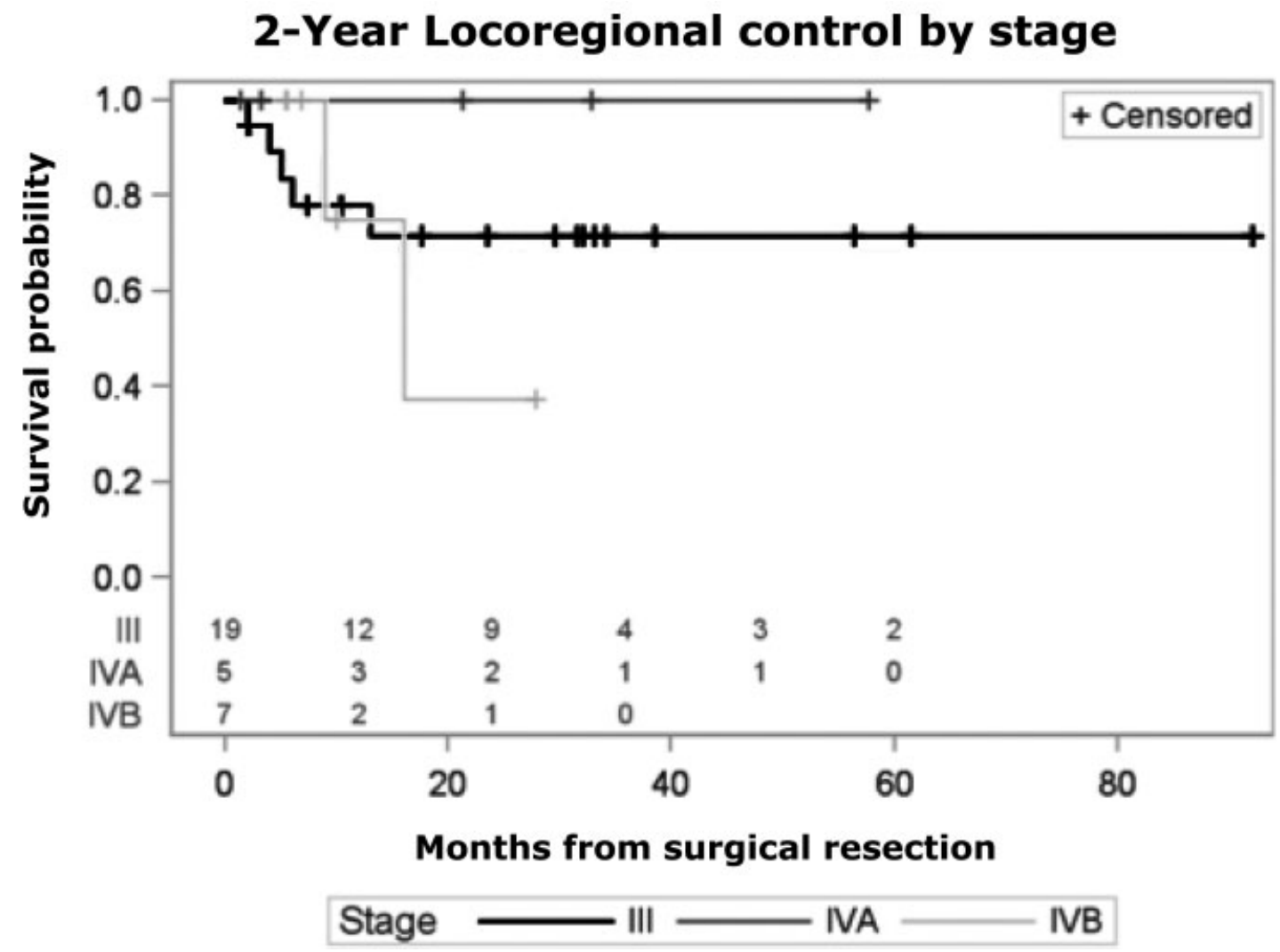

Fig. 3 2-year locoregional control by stage. 


\section{2-Year distant control by stage}

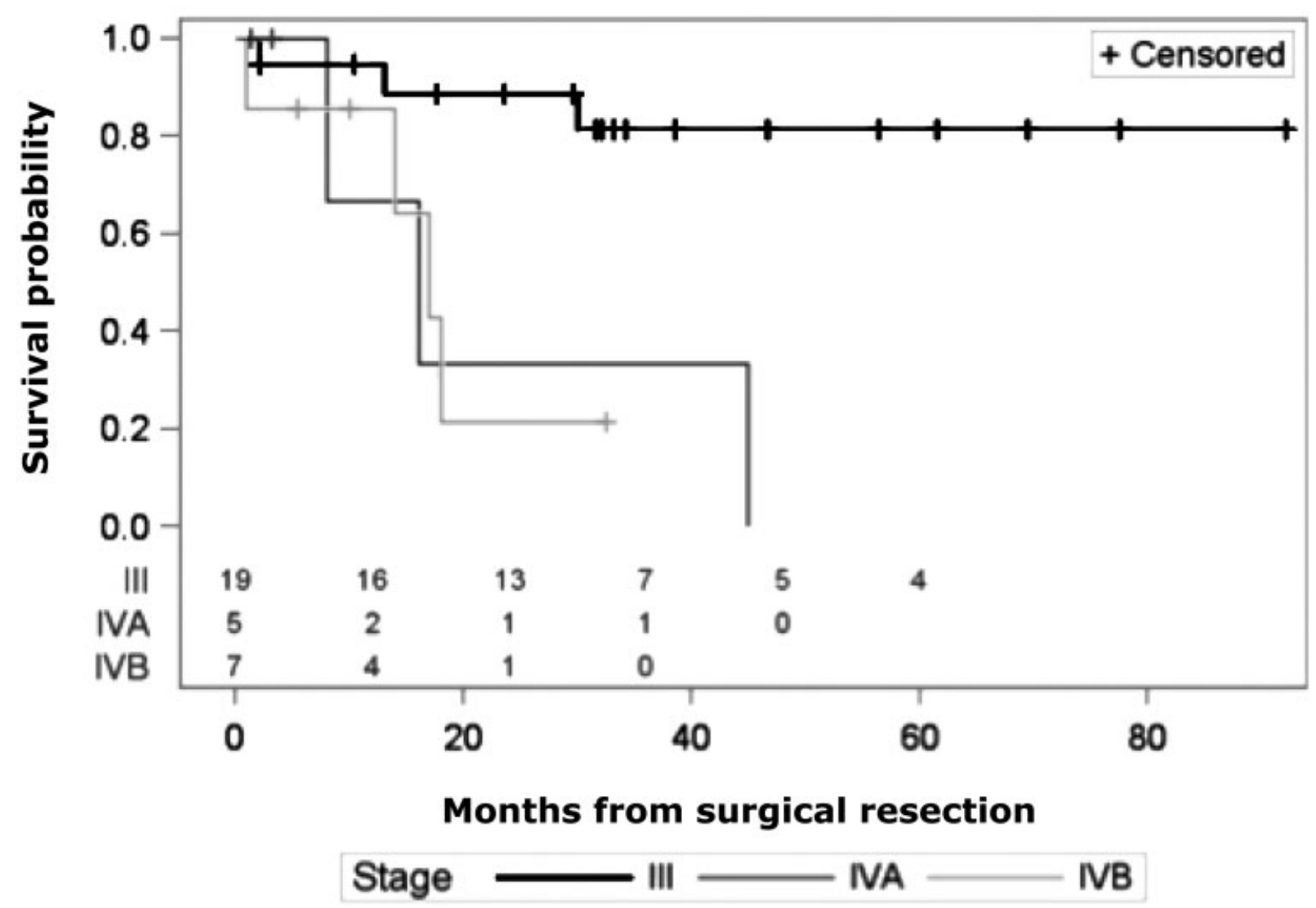

Fig. 4 2-year distant control by stage.

disease over a 3.5-year period and experienced just a short term mild skin rash as a side effect. The second patient had T4aN0M0 mucosal melanoma of the nasal cavity. This patient underwent a combined surgical approach followed by adjuvant radiation therapy. They developed distant disease 4 years following treatment, with metastases to the pancreas, liver, and small bowel. The patient was initiated on pembrolizumab therapy at the time of diagnosis of distant disease. They have remained with stable disease over a 1.5year period, having experienced vitiligo as a side effect of treatment. Our third patient had T4bNOM0 mucosal melanoma of the ethmoid sinus. They underwent endoscopic resection but developed abdominal metastatic disease within 1 month postsurgery and thus did not receive adjuvant radiation therapy. The patient died of disease 4 months

Table 6 2-year Kaplan-Meier estimates (95\% Cl) of time to event outcomes

\begin{tabular}{|l|l|l|l|}
\hline & $\begin{array}{l}\text { Stage III } \\
\boldsymbol{n}=\mathbf{1 9}\end{array}$ & $\begin{array}{l}\text { Stage IVA } \\
\boldsymbol{n}=\mathbf{5}\end{array}$ & $\begin{array}{l}\text { Stage IVB } \\
\boldsymbol{n}=\mathbf{7}\end{array}$ \\
\hline OS & $87 \%(58.97)$ & $67 \%(5.95)$ & $57 \%(17.84)$ \\
\hline LC & $72 \%(44.87)$ & $100 \%(\mathrm{Cl}$ not estimable) & $38 \%(1.81)$ \\
\hline DC & $89 \%(62.97)$ & $33 \%(1.77)$ & $21 \%(1.60)$ \\
\hline
\end{tabular}

Abbreviation: $\mathrm{Cl}$, confidence interval. after initiation of ipilimumab. Patient characteristics are displayed in - Table 8.

\section{Discussion}

Sinonasal melanoma remains a challenging disease entity with a poor prognosis. Some patients experience a prolonged survival despite multiple local recurrences or development of distant metastatic disease, whereas others rapidly decline despite negative-margin surgical resection and adjuvant radiation therapy. This suggests the presence of underlying differences in genetic makeup or epigenetic factors (e.g., immune response) between these tumors which have not yet been defined.

Management of sinonasal melanoma at our institution has largely transitioned from the utilization of open approaches to those including endoscopic alone or combined approaches. All disease stages have been successfully managed endoscopically. The decision to proceed with an endoscopic approach has been made based on clinical and radiographic extent of disease, patient comorbidities, and surgeon experience. With continued practice and technique development, we are able to further push the boundaries of disease we are able to approach endoscopically while minimizing morbidity at our institution. Overall survival at 2 years for stage III disease is $87 \%$ at our practice. This sharply declined to $57 \%$ in more advanced stage IVB disease. Locoregional control for stage III disease is $72 \%$. We 
Table 7 Hazard ratios and p-values from Cox's proportional hazards models for overall survival (OS), locoregional control (LC), and distant control (DC)

\begin{tabular}{|l|l|l|l|}
\hline & OS & LC & DC \\
\hline Age & $0.97(0.91,1.03), p=0.34$ & $0.98(0.91,1.06), p=0.59$ & $0.97(0.91,1.04), p=0.37$ \\
\hline Sex (female vs. male) & $1.61(0.35,7.49), p=0.54$ & $2.41(0.29,20.07), p=0.41$ & $1.68(0.36,7.93), p=0.51$ \\
\hline Stage IVA vs. III & $2.27(0.44,11.78), p=0.33$ & $\mathrm{a}$ & $7.65(1.51,38.71), p=0.01$ \\
\hline Stage IVB vs. III & $3.87(1.02,14.74), p=0.047$ & $1.31(0.25,6.87), p=0.75$ & $7.38(1.56,34.84), p=0.01$ \\
\hline Stage IVA vs. IVB & $1.70(0.31,9.47), p=0.54$ & $\mathrm{a}$ & $0.96(0.20,4.58), p=0.96$ \\
\hline $\begin{array}{l}\text { Primary site } \\
\text { (sinus vs. nasal cavity) }\end{array}$ & $2.84(0.82,9.88), p=0.10$ & $0.52(0.06,4.33), p=0.54$ & $6.06(1.58,23.27), p=0.009$ \\
\hline Smoking (Y vs. N) & $1.86(0.53,6.51), p=0.33$ & $0.33(0.04,2.76), p=0.31$ & $0.57(0.12,2.70), p=0.48$ \\
\hline Radiation (Y vs. N) & $0.76(0.20,2.89), p=0.69$ & $0.63(0.12,3.26), p=0.58$ & $2.78(0.35,22.03), p=0.33$ \\
\hline Margins (pos vs. neg) & $1.32(0.35,4.99), p=0.68$ & $\mathrm{~b}$ & $0.94(0.20,4.74), p=0.94$ \\
\hline D from diagnosis to OR & $0.99(0.96,1.02), p=0.35$ & $0.99(0.95,1.02), p=0.44$ & $0.99(9.95,1.02), p=0.40$ \\
\hline
\end{tabular}

Abbreviations: N, no; neg, negative; OR, operation; pos, positive; Y, yes.

${ }^{a}$ No local recurrences in stage IVA

${ }^{\mathrm{b}}$ No positive margins with local recurrence.

Table 8 Characteristics of patients receiving immunotherapy

\begin{tabular}{|c|c|c|c|c|c|c|c|c|c|}
\hline Patient & Stage & $\begin{array}{l}\text { Primary } \\
\text { stage }\end{array}$ & $\begin{array}{l}\text { Date of } \\
\text { diagnosis }\end{array}$ & $\begin{array}{l}\text { Date of } \\
\text { surgery }\end{array}$ & Technique & $\begin{array}{l}\text { Adjuvant } \\
\text { XRT }\end{array}$ & Metastatic disease & Immunotherapy & Status \\
\hline 1 & T4bNOMO & Ethmoid & $5 / 18 / 2011$ & $7 / 6 / 2011$ & open & yes & $\begin{array}{l}\text { November 2012: neck; } \\
\text { December 2014: pelvis; } \\
\text { May 2014: temporal lobe }\end{array}$ & $\begin{array}{l}\text { Ipilimumba, } \\
\text { nivolumab }\end{array}$ & $\begin{array}{l}\text { Alive last RV } \\
12 / 4 / 2017\end{array}$ \\
\hline 2 & T4aNOM0 & $\begin{array}{l}\text { Nasal } \\
\text { cavity }\end{array}$ & $5 / 29 / 2012$ & $7 / 20 / 2012$ & combined & yes & $\begin{array}{l}\text { April 2016: pancreas, } \\
\text { liver; September 2016: } \\
\text { small bowel }\end{array}$ & Pembrolizumab & $\begin{array}{l}\text { Alive last RV } \\
1 / 30 / 2018\end{array}$ \\
\hline 3 & T4bN0M0 & Ethmoid & $1 / 10 / 2013$ & $1 / 25 / 2013$ & endoscopic & no & February 2013: abdomen & Ipilimumab & $\begin{array}{l}\text { Deceased } \\
6 / 26 / 2013\end{array}$ \\
\hline
\end{tabular}

Abbreviations: RV, return visit; XRT, radiation.

did not have any locoregional recurrences in our stage IVA subgroup, though there were only five patients included, all of which were received adjuvant radiation therapy. The small sample size significantly limits our ability to interpret this information. Locoregional control was notably worse in the stage IVB patients at $38 \%$. As expected, the distant control declined with increasing stage.

It's difficult to make conclusions on why our disease control is higher than the average reported given our small sample size. Our overall approach includes aggressive, margin-negative, and surgical resection followed by adjuvant radiation therapy in the majority of cases. We also utilize intraoperative, frozen section, and margin analysis. Though frozen section analysis for melanoma is not standard and margins are sometimes overturned on final pathology, we do feel this additional information helps to guide margin-negative resection.

Most of our patients underwent adjuvant radiation therapy. The few that did not either had minimal disease burden or progressed rapidly to distant disease and death without time for adjuvant intervention. Given the poor prognosis associated with this disease and the possibility for skip lesions despite 'negative' surgical margins within the sinonasal cavity, it is our practice to routinely recommend adjuvant radiation therapy. ${ }^{17}$ We agree with the majority of authors in considering adjuvant radiotherapy to increase local and regional control even though it does not increase survival independently of tumor stage. ${ }^{18-23}$

The management of patients with unresectable and metastatic disease remains a challenge. Outcomes and management of these patients tend to parallel those of advanced cutaneous melanoma patients. To date, there have been no published randomized controlled trials evaluating the efficacy of newer immunologic therapies in the setting of sinonasal melanoma. Several retrospective studies have reviewed early outcomes in this patient cohort with encouraging results. The low incidence rate of this disease makes the development of randomized trials unlikely to occur, and use of these agents for sinonasal melanoma is predicated on the understanding of the outcomes in cutaneous melanoma and small retrospective reviews. Postow et al reviewed the efficacy of ipilimumab for patients with metastatic or unresectable mucosal melanoma and showed that although the overall response rate was low, some patients can achieve durable responses with a reasonable side effect profile. ${ }^{17}$ Of note, only 12 of the 33 patients analyzed had a disease origin within the sinonasal cavity. We plan to continue gathering data on our patient cohort in hopes to provide some insight into the efficacy of immunotherapy in the setting of advanced sinonasal melanoma. 
Our study is limited by the small sample size. Sinonasal melanoma is a rare disease, making a single institution review of outcomes difficult, even at a large academic center. This limits our ability to perform multivariate analysis on a large number of clinical and pathologic characteristics. With a small sample size, is it possible that some factors influencing our outcomes are not identified due a lack of power.

\section{Conclusion}

Treatment of sinonasal melanoma has drastically changed over the past decade with the advent of expanded endonasal techniques. At our institution, all disease stages have been successfully managed endoscopically and the decision to proceed with this approach is made carefully by considering clinical and radiographic extent of disease, patient comorbidities, and surgeon experience. Management of advanced and metastatic disease is also a changing paradigm with the introduction of immunotherapy. Here we present a case report of three patients, two of which have sustained stable metastatic disease burden on immunotherapy with well over a year of follow-up and with minimal side effects.

\section{Conflict of Interest}

None declared.

\section{References}

1 Chang AE, Karnell LH, Menck HR; The American College of Surgeons Commission on Cancer and the American Cancer Society. The National Cancer Data Base report on cutaneous and noncutaneous melanoma: a summary of 84,836 cases from the past decade. Cancer 1998;83(08):1664-1678

2 Shah JP, Huvos AG, Strong EW. Mucosal melanomas of the head and neck. Am J Surg 1977;134(04):531-535

3 Prasad ML, Patel S, Hoshaw-Woodard S, et al. Prognostic factors for malignant melanoma of the squamous mucosa of the head and neck. Am J Surg Pathol 2002;26(07):883-892

4 Lund VJ, Howard DJ, Harding L, Wei WI. Management options and survival in malignant melanoma of the sinonasal mucosa. Laryngoscope 1999;109(2 Pt 1):208-211

5 Gaze MN, Kerr GR, Smyth JF; The Scottish Melanoma Group. Mucosal melanomas of the head and neck: The Scottish experience. Clin Oncol (R Coll Radiol) 1990;2(05):277-283

6 Stern SJ, Guillamondegui OM. Mucosal melanoma of the head and neck. Head Neck 1991;13(01):22-27

7 Suh JD, Ramakrishnan VR, Chi JJ, Palmer JN, Chiu AG. Outcomes and complications of endoscopic approaches for malignancies of the paranasal sinuses and anterior skull base. Ann Otol Rhinol Laryngol 2013;122(01):54-59

8 Nicolai P, Battaglia P, Bignami M, et al. Endoscopic surgery for malignant tumors of the sinonasal tract and adjacent skull base: a 10-year experience. Am J Rhinol 2008;22(03):308-316

9 Lund V. Malignant melanoma of the nasal cavity and paranasal sinuses. J Laryngol Otol 1982;96(04):347-355

10 Hodi FS, O'Day SJ, McDermott DF, et al. Improved survival with ipilimumab in patients with metastatic melanoma. N Engl J Med 2010;363(08):711-723

11 Robert C, Thomas L, Bondarenko I, et al. Ipilimumab plus dacarbazine for previously untreated metastatic melanoma. N Engl J Med 2011;364(26):2517-2526

12 Robert C, Long GV, Brady B, et al. Nivolumab in previously untreated melanoma without BRAF mutation. N Engl J Med 2015;372(04):320-330

13 Weber JS, D'Angelo SP, Minor D, et al. Nivolumab versus chemotherapy in patients with advanced melanoma who progressed after anti-CTLA-4 treatment (CheckMate 037): a randomised, controlled, open-label, phase 3 trial. Lancet Oncol 2015;16(04): 375-384

14 Dummer R, Daud A, Puzanov I, et al. A randomized controlled comparison of pembrolizumab and chemotherapy in patients with ipilimumab-refractory melanoma. J Transl Med 2015;13 (Suppl 1:05

15 Larkin J, Chiarion-Sileni V, Gonzalez R, et al. Combined Nivolumab and Ipilimumab or monotherapy in untreated melanoma. N Engl J Med 2015;373(01):23-34

16 Postow MA, Luke JJ, Bluth MJ, et al. Ipilimumab for patients with advanced mucosal melanoma. Oncologist 2013;18(06):726-732

17 Postow MA, Hamid O, Carvajal RD. Mucosal melanoma: pathogenesis, clinical behavior, and management. Curr Oncol Rep 2012; 14(05):441-448

18 Moreno MA, Roberts DB, Kupferman ME, et al. Mucosal melanoma of the nose and paranasal sinuses, a contemporary experience from the M. D. Anderson cancer center. Cancer 2010;116(09): 2215-2223

19 Benlyazid A, Thariat J, Temam S, et al. Postoperative radiotherapy in head and neck mucosal melanoma: a GETTEC study. Arch Otolaryngol Head Neck Surg 2010;136(12):1219-1225

20 Shiga K, Ogawa T, Kobayashi T, et al. Malignant melanoma of the head and neck: a multi-institutional retrospective analysis of cases in northern Japan. Head Neck 2012;34(11):1537-1541

21 Temam S, Mamelle G, Marandas P, et al. Postoperative radiotherapy for primary mucosal melanoma of the head and neck. Cancer 2005;103(02):313-319

22 Krengli M, Jereczek-Fossa BA, Kaanders JH, Masini L, Beldì D, Orecchia $\mathrm{R}$. What is the role of radiotherapy in the treatment of mucosal melanoma of the head and neck? Crit Rev Oncol Hematol 2008;65(02):121-128

23 Bachar G, Loh KS, O'Sullivan B, et al. Mucosal melanomas of the head and neck: experience of the Princess Margaret Hospital. Head Neck 2008;30(10):1325-1331 